\title{
IMPROVING THE QUALITY OF LIFE IN CLIMAX WITH KINETOTHERAPY AND NATURAL FACTORS IN VATRA DORNEI RESORT
}

\author{
Maria Daniela Crăciun \\ University "Stefan cel Mare" Suceava \\ daniela_mariacr@yahoo.com
}

\begin{abstract}
Introduction

Menopause is a physiological period of a woman's life accompanied by multiple symptoms that affect quality of life. Functional disorders caused by menopause may benefit from prophylactic treatment in Vatra Dornei resort to rebalance the body.
\end{abstract}

\section{Material and Methods}

The study was conducted between July10, 2014 - September 15, 2014 on a group of 15 women, who received specific kinetic and resort treatment for 18 days, being individualized based on personal history. Evaluation of patients was done at beginning of the treatment the day $10^{\text {th }}$ of July, and the last day of treatment the day $15^{\text {th }}$ of September, recording pain score on VAS scale, the score for quality of life WHQ, and 10 parameters characteristic of menopause. The treatment with natural factors applied in Vatra Dornei resort consisted of the following procedures: mountain landscaped climbing paths, massage, kinetotherapy, general warm baths with peat mud, plants, carbonated mineral spring waters, electrotherapy, crenotherapy.

\section{Results:}

Age patients monitored ranged between 40 and 63 years with an average $50.67( \pm$ 8.07). At the onset of this treatment pain average value was $3.93( \pm 1.48)$ and at the final the average value of only $1.13( \pm 0.74)$. After applying balneo-physio-kinetic treatment it was observed an improvement of the average of the 10 tested parameters.

At the beginning of the treatment WHQ index shows an impairment of quality of life on average $56 \%$, after 10 days $31 \%$ and by the end of treatment only $14 \%$.

\section{Conclusions:}

After the application of the treatment with natural balneo-kineto-therapeutical factors of Vatra-Dornei resort, there is a significant improvement in specific climax disorders.

Keywords: menopause, climax, natural factors, kinetotherapy, Vatra Dornei. 


\section{Introduction}

Menopause is a physiological period of a woman's life accompanied by multiple symptoms that affect quality of life.

Menopause occurs around the age of 50, and with the natural cessation of the menstrual cycle are some neurodegenerative disorders: hot flushes followed by waves of sweating, heart palpitations (specific for people who are anxious, nervous), irregular periods with very heavy bleeding, bone rarefaction (osteoporosis), emotional disorders (nervousness, fear or depression), exacerbation of appetite, frequent urination, irritability, difficulty concentrating, insomnia and depression(1).

Because of these major hormonal changes in a woman's body may occur or worsen some diseases in which the body is prone: high blood pressure, obesity, neurosis.

A woman in menopause, can lead an active life, existing symptoms may be alleviated by medical and natural solutions(2).

Drug therapy significantly improves symptoms of hormone replacement in climax but is not too accepted due to the side effects: headache, weight gain, seborrhea, breast tenderness, amplifying the risk for ovarian cancer, cervical cancer, breast cancer(3).

As a result new methods of treatment were sought to improve quality of life of women in this stage of their lives.

Typical menopausal disorders benefit from natural factors acting the balance in establishing recommendations and taking into account coexisting disorders: rheumatism, cardiac, digestive or respiratory.

Due to reactivity neurotic type are indicated resorts with sedative neutral bioclimate climate and mountain climate (700-800 m, altitude) which are characterized by monthly average air temperature, atmospheric pressure and moderate duration of sunshine throughout the year.

Vatra Dornei is a resort in Suceava County and has a tonic to sedative-neutral mountain climate, ferruginous carbonated mineral spring waters and peat mud.

The treatment options available: warm baths with carbonated mineral spring waters in the bathtubs, mofettes, warm mud applications, hydrothermotherapy, electrotherapy, kinetotherapy, aerosol-therapy, mechanictherapy, mountain landscaped climbing paths; the resort Vatra Dornei is indicated for cardiovascular diseases, diseases such as rheumatic musculoskeletal disorders, disorders of the functional climax, neurosis(4).

Functional genital disorders caused by menopause are functional overactive disorders and can benefit of resort treatment with prophylactic role meant to rebalance the body.

\section{Material and methods}

Given the complexity and variability of symptoms of climax, I selected 15 postmenopausal women presenting with a clinical characteristic picture.

The study was conducted in Vatra Dornei resort, in the location of Complex “'Intus”, during the period July 10, 2014 September 15, 2014.

The selected patients have received specific kinetic and resort treatment for 18 days, being individualized, based on personal history.

Patients have accused mainly headache, dizziness, insomnia, nervousness, anxiety, depression, hot flashes, however altering the quality of life and social integration capacity.

Evaluation of patients was done at the beginning of treatment in the $10^{\text {th }}$ day and in the last day of treatment, recording pain score on VAS - scale, WHQ (Women's Health Questionnaire) for quality of life and 10 parameters characteristic of menopause(5). 
Treatment with natural factors applied in Vatra Dornei resort consisted of the following types of procedures in different combinations and based on patient tolerability:

- Daily walking on mountain landscaped climbing paths with a low or medium altitude based on the patients physical conditions, having the goal to improve the effort capacity of the cardiovascular and respiratory systems, increase of neuromuscular tonus, improving of venous and arterial circulation, increase of lipids metabolism. Also next to these, the climate contributes to put at rest the disturbed neuralvegetative and endocrine functions caused by menopause.

- General massage with sedative role, to balance the neuralvegetative system which increases trophicity and improves circulation;

- Kinetotherapy to improve the effort capacity and cardio-respiratory functions, decrease of pain, stress relieve, osteoporosis prevention and improve the metabolic processes and so to minimize the Domino effect $(6,7)$;

- General warm baths with peat mud or plants to increase the peripheral circulation and metabolic processes, stress relieve and insomnia, inducing general relaxation.

- Carbonated mineral spring waters baths act over the body through the three components: thermal (water temperature), mechanical (hydrostatic pressure and bubbles), and chemical (through chemical composition of carbonated mineral spring waters). Through their peripheral vasodilator effect, the carbonated mineral spring waters contribute improving the cardiovascular system and rebalance the neural-vegetative and mental functions(4).

- The physical methods used as helping in menopausal dysfunctions contribute to rebalance the over reactive neuralvegetative functions in this stage of woman life. Some of the following methods are: electrotherapy of low and medium frequency for their vassal-active, antispasmodic and sedative effect, thermotherapy with wax applications, light baths and shortwave diathermy $(1,3)$.

- Crenotherapy uses carbonated spring mineral waters-ferruginous, sodium, calcium, magnesium and hypotonic types; specific natural factors of the Vatra Dornei resort with beneficial effects on the cardiovascular and digestive systems influence the dynamics of blood circulation with minimal self energy consumption.

\section{Results}

Age patients monitored ranged between 40 and 63 years with an average 50.67 ( \pm 8.07) majority being in the premenopausal stage and the near menopausal stage, and only 4 women being menopausal.

Specific symptoms of menopause in women tested has a mean of 2.90 ( \pm 2.03) altered a maximum of seven years and a minimum of 0.5 years.

Most patients present rheumatic and heart disease and related disorders in the climax, so that's why I evaluated and pain VAS (Visual Analog Scale). 


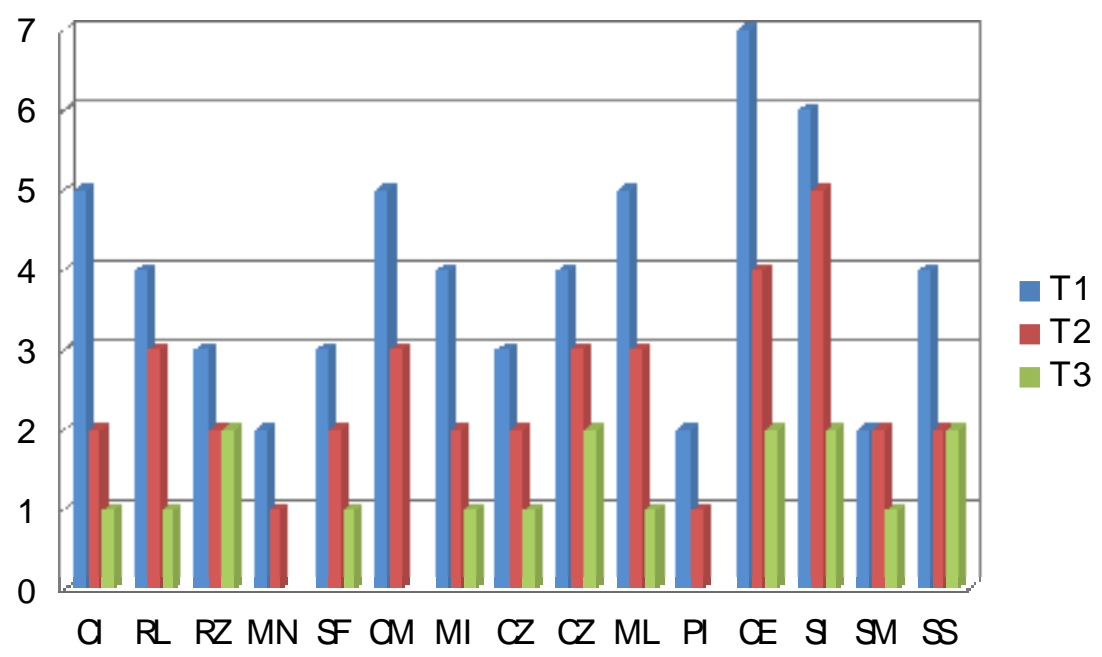

Figure 1-Dynamics of VAS (pain scale) on tested patients.

At the onset of this treatment the pain averages shows the average $3.93( \pm 1.48)$ and at the end of treatment there was a significant reduction of pain, the average value of only $1.13( \pm 0.74)$.

The following complains of the main group were analyzed during the course of treatment: insomnia, anxiety, depression, palpitations, nervousness/ stress, headache, back pain, hot flushes, bloating, difficulty concentrating.

After the balneo-physical-kinetic therapies treatments applications an improvement average and tested parameters as shown in the following table:

Table 1- Test parameters during treatment with evolution

\begin{tabular}{|l|l|l|l|l|}
\hline $\begin{array}{l}\text { No. } \\
\text { crt }\end{array}$ & Parameters tested & Testing 1 & Testing 2 & Testing 3 \\
\hline $\mathbf{1}$ & Insomnia & $2.36( \pm 1.03)$ & $2.86( \pm 0.69)$ & $3.50( \pm 0.50)$ \\
$\mathbf{2}$ & Anxiety & $2.57( \pm 0,99)$ & $3.14( \pm 0.85)$ & $3.71( \pm 0.46)$ \\
$\mathbf{3}$ & Depression & $2.79( \pm 0,80)$ & $3.21( \pm 0.43)$ & $3.71( \pm 0.46)$ \\
$\mathbf{4}$ & Palpitations & $2.71( \pm 0,75)$ & $3.50( \pm 0.75)$ & $3.79( \pm 0.43)$ \\
$\mathbf{5}$ & Nervousness/ stress & $2.36( \pm 0,92)$ & $3.07( \pm 0.65)$ & $3.57( \pm 0.49)$ \\
$\mathbf{6}$ & Headache & $2.14( \pm 0,78)$ & $3.07( \pm 0.65)$ & $3.71( \pm 0.46)$ \\
$\mathbf{7}$ & Backpain & $1.79( \pm 0,72)$ & $2.93( \pm 0.55)$ & $3.79( \pm 0.43)$ \\
$\mathbf{8}$ & Hot flashes & $1.79( \pm 0,72)$ & $3.14( \pm 0.59)$ & $3.43( \pm 0.49)$ \\
$\mathbf{9}$ & Bloating & $2.86( \pm 0,69)$ & $3.29( \pm 0.66)$ & $3.50( \pm 0.50)$ \\
$\mathbf{1 0}$ & Difficulty & $2.07( \pm 065)$ & $3.29( \pm 0.46)$ & $3.79( \pm 0.43)$ \\
& concentrating & & & \\
\hline
\end{tabular}

To get as much information about mental and physical health status of patients the questionnaire WHQ was applied, which included 36 questions about mental and physical health of postmenopausal women.

At the beginning of the treatment quality of life was altered on average $56 \%$, $31 \%$ after 10 days and at the end of treatment only $14 \%$, which indicates that Vatra Dornei resort natural factors have a beneficial effect for reducing specific symptoms of climax contributing to autonomic regulation and neuropsychological rebalance. 
T3

$\mathrm{T} 2$

INDICEEWHQ

$\mathrm{T} 1$

$\begin{array}{lllllll}0 & 10 & 20 & 30 & 40 & 50 & 60\end{array}$

Figure.2-Dynamics VAS pain patients tested

\section{Discussion}

World Health Organization statistics showed that in some Asian countries Japan, Indonesia, Korea, menopausal disorders are rarer (8).

All the studies made in the last twenty years shows that menopausal treatments carried out against disorders must be associated with a special diet with many grains, vegetables, raw fruits, completely eliminating stimulants, alcohol and coffee, and food additives which disrupt the hormonal balance and on the other hand may have undesirable interactions with the treatments performed.

Women who practice regular physical activity report a better quality of life. There are not significant differences of disorders between women who exercise and those that are not (6).

Women who are overweight complain more pronounced vasomotor symptoms compared with those with a normal weight regardless of their origin (8).

The middle-aged women are active at low to medium intensity, doing exercise once a week or are sedentary, so the climax disorders are so strong; it is well known that by practicing regular physical exercises endorphins are induced and so contributing at psycho-somatic wellbeing.
A pilot study done at the University Hospital of Linkping, Sweden, has correlated breathing relaxation exercises through a powerful program for women with menopausal disorders, especially hot flashes, sweating and cardiac arrhythmia and the participants achieved a remarkable reduction of $73 \%$ of hot flashes after six months.(6)

In the study $53.33 \%$ of selected women aged between 40-50ani and $46.66 \%$ were aged 50-60 years, which showed that a woman in menopause is not old, but young, being able to lead an active life in all respects.

Genital functional disorders caused by menopause are influenced by the intensity of psychiatric symptoms, knowing that the psycho-emotional problems can cause some physical disorders.

Currently, promoting the health of women in the demographic politics and increasing social role of women, the treatment of gynecological diseases through balneo-physio-kinetotherapeutical methods gets increasingly higher attention. 


\section{Conclusions:}

The treatment of disorders of the climax is good to start with natural remedies in various forms: the resort cure by specific natural factors profile, physical therapy, phytotherapy there are most often favorable responses.

After balneo-kinetic treatments applications with natural factors of VatraDornei resort there is a significant improvement in the parameters tested.

Pain associated with collateral diseases decreased after the first day of treatment, its perception being influenced and by specific menopausal disorders.

It also noted an increase in psychological tonus of the women who were treated and the disappearing of nervousness and anxiety.

The resort of Vatra Dornei is not specific in functional disorders of menopause but natural factors of this mountain region have beneficial effects for reducing symptoms of climax.

The complex treatment of women under this special period led to an increase in quality of life that favors a normal social activity

\section{BIBLIOGRAPHY:}

1. Stephenson R. , O Connor L., Obstetric and Gynecologic care in physical therapy, second edition, Slack Incorporated, 2000,3: 73-92

2. Gheorghiu I., Gheorghiu A., Rusu M., Tratamente natural, Ed. Minerva, Bucureşti 1993;

3. Irion Jean M., Irion Glenn Women's Health in Physical Therapy Wolters Kluwer Health/Lippincott Williams \& Wilkins, 2010, 4: 460-473

4. Berlescu E., Mică enciclopedie de balneoclimatologie a României, Ed. Medicală, Bucuresti, 1996: 145, 221, 229-231;

5. Hunter M. The women's health questionnaire: a measure of mid-aged women's perceptions of their emotional and physical health. Psychol Health. 1992;7:45-54.

6. Daley A., Mac Artur C., StokesLampard H., et all, Exercise participation, body mass index, and health-related quality of life in women of menopausal age, $\mathrm{Br} J$ Gen Pract. 2007, 1, 57 (535): 130-135;

7. Mirzaiinjmabadi $K$, Anderson D, Barnes $M$. The relationship between exercise, body mass index and menopausal symptoms in midlife Australian women. Int J Nurs Pract. 2006;12(1):28-34

8. Palacios S., Henderson $V . \quad W$., Siseles N., Tan D., Villaseca P., Age of menopause and impact of climacteric symptoms by geographical region, Climacteric, 2010; 13: 419-428; 